\title{
The Relevance of Fractional Amplitude of Low-Frequency Fluctuation to Interference Effect
}

\author{
Yuqin Deng ${ }^{\mathrm{a}}$, Yan Wang ${ }^{\mathrm{b}}$, Xiaoqian Ding ${ }^{\mathrm{c}}$, Yi-Yuan Tang ${ }^{\mathrm{d} *}$ \\ ${ }^{a}$ Department of Physics, Institute of Neuroinformatics, Dalian University of
} Technology, Dalian 116024, Liaoning Province, China.

${ }^{\mathrm{b}}$ Interdisciplinary Center for Social and Behavioral Studies, Dongbei University of Finance and Economics, Dalian 116024, Liaoning Province, China.

${ }^{\mathrm{c}}$ Centre for Psychological Health Education, Dalian Nationalities University, Dalian 116024, Liaoning Province, China.

${ }^{\mathrm{d}}$ Department of Psychological Sciences, Texas Tech University, Lubbock, Texas, United States of America

*Corresponding author

Tel.: +1-806-742-3711

Email address: yiyuan.tang@ttu.edu 


\section{Abstract}

Growing evidence has indicated a potential connection between resting-state functional magnetic resonance imaging (RS-fMRI) signal and cognitive performance. However, the relationship between intrinsic neural activity and behavioral interference effect on cognitive control has been poorly understood. In the present study, seventy-eight healthy subjects underwent RS-fMRI and performed Multi-Source Interference Task (MSIT). The fractional amplitude of low-frequency fluctuation (fALFF) was measured as an indicator of intrinsic brain activity. The difference in reaction times between interference and control conditions in MSIT was evaluated as interference effect. Then we examined the associations between fALFF and interference effect using partial correlation analysis controlling for age, gender and mean framewise displacement. The results demonstrated that fALFF values in orbital prefrontal cortex (OPFC) and right inferior frontal cortex (IFC) were negatively correlated with the interference effect in MSIT. The findings manifest that OPFC and right IFC may influence the processing efficiency of cognitive conflict and play a crucial role in cognitive control.

\section{Key words}

Resting-state functional magnetic resonance imaging, Fractional amplitude of low-frequency fluctuation, Multi-source interference task, Interference effect 


\section{Introduction}

In the era of information explosion, people are exposed to vast amount of relevant and irrelevant information. To enhance efficiency, it is crucial for people to effectively distinguish useful information from the rest. For example, if one desires to use the web to access the most efficacious information on one's research, special attention is demanded to disregard irrelevant information (such as latest breaking current news) from inconsequential websites and fully concentrate on the most relevant websites. Generally speaking, the ability to effectively eliminate interference from extraneous information and predominantly focus on the appropriate information could be extremely helpful in our daily life. To better explore the psychological processes for such phenomenon, interference resolution tasks on cognitive control are widely applied [1].

Among these interference resolution tasks, Multi-Source Interference Task (MSIT), which combined the Stroop, Simon and flanker effects, has been extensively studied. The initial studies on MSIT have showed that MSIT could robustly induce the activation in the cingulo-frontal-parietal network in both group and individual analyses [2-3]. The cingulo-frontal-parietal network (CFP network) encompasses dorsal anterior cingulate cortex (dACC), dorsolateral prefrontal cortex (dIPFC) and superior sections of parietal cortex. The activation of dACC ascribes to such relevant processes as the identification of the target, novelty or error, response choice, task difficulty, stimulus/response conflict and the regulation of ongoing behavioral adjustment [2-5].The dlPFC and superior sections of parietal cortex are responsible 
for initiating and modulating the cognitive control $[3,6,7]$. The MSIT-based functional imaging studies have been widely used to investigate the activation of dACC or CFP network in a wide range of population, including both clinical (such as schizophrenia, obsessive-compulsive disorder (OCD), attention-deficit/hyperactivity disorder, cannabis users, depression, etc.) [8-16] and nonclinical samples (such as children, adolescents, youth, old participants, female twins, etc.) [17-20]. Moreover, some studies have examined the connections between MSIT-evoked activation and resting-state. For example, Fitzgerald et al. found that pediatric OCD displayed hyperactivation of $\mathrm{dACC}$, which showed different patterns of functional connectivity during MSIT-related condition and during resting state condition [12]. Davey et al. found that resting functional connectivity of the subgenual anterior cingulate cortex showed connection with different regions in response to MSIT in depression and healthy control groups [16]. In the latest study, when the researchers measured teenage brain activities during MSIT and during a resting-state, they observed that the brain network of teenage cognitive control was characterized by a dynamic and reciprocity between the cognitive control network and default-mode network [20]. These results reflect that the resting state is correlated with MSIT-evoked activation and may characterize the behavioral relevancy of spontaneous neural activity. To further characterize the inter-individual differences in MSIT, it is necessary to recognize how these differences associate with alternations in the brain's intrinsic activity.

Resting-state functional magnetic resonance imaging (RS-fMRI) is an important $-4-$ 
tool for investigating and characterizing the spontaneous neural activity in the absent of any specific cognitive tasks [21]. Relative to the resting state, task-evoked elevations of the brain energy expenditure are little (less than 5\%), suggesting that the brain energy expenditure is less influenced by task execution but more by intrinsic activity $[22,23]$. Correspondingly, the resting state approach is quite suitable for understanding the essential feature of brain function [22, 23].The spontaneous low-frequency (0.01-0.08 Hz) oscillations (LFO) have been corroborated to reveal the intrinsic neuronal activity. The fractional amplitude of low-frequency fluctuation (fALFF) is a normalized measure of amplitude of low-frequency fluctuation (ALFF) [24] and is one of the important indexes in reflecting the LFO fluctuations of RS-fMRI signals. As a robust and reliable method in RS-fMRI, fALFF measure could effectively suppress the physiological artifact $[24,25]$. Some recent studies have exhibited that the inter-subject differences of fALFF have identified tight linkages with individual variance in personality traits and multiple cognitive tasks [26-31]. The four of the Big Five personality traits (extraversion, neuroticism, openness and conscientiousness) were shown to correlate with the fALFF in different cerebral areas [26]. Wei et al. further found that the associations between fALFF and extraversion or neuroticism dimensions relied on distinct frequency bands [27]. fALFF was shown to be associated with task-relevant brain activity and with behavior performance during an Eriksen flanker task [28]. Meanwhile, fALFF in the supplementary motor area (SMA) and pre-SMA was reported to be negatively linked to not only age, but also the stop signal reaction time (SSRT) in the stop signal task [29]. More recently, van 
Dam et al. demonstrated that various memory measures, including general, domain-specific and demand-specific memory abilities, were related to fALFF in the extensive and distinct brain networks [30]. The positive correlation between fALFF and reading skill was found in the left precentral gyrus and superior temporal plane, whose regions served a role in phonological processing of language [31]. All the investigated correlations reveal that fALFF, as a data-driven method, is appropriate to exploratory analysis and the correlation analyses between fALFF- and behavior could be valuable gauges to examine the potential neural characteristics for cognitive control.

However, to our best knowledge, no study has explored the relationship between behavioral results of interference effect on MSIT and spontaneous brain activity. Therefore, the current study aims to explore how interference effect of MSIT is supported by intrinsic neural activity without performing any cognitive task. We focused on the relationship between fALFF and interference effect of MSIT and investigated the neural basis of individual differences by measuring fALFF of RS-fMRI signals. The interference effects were estimated by subjects' behavior performance in MSIT. Based on the previous imaging study on MSIT, we predicted that the regions related to cognitive control would show significant fALFF-interference effect correlations.

\section{Methods}

\subsection{Participants}

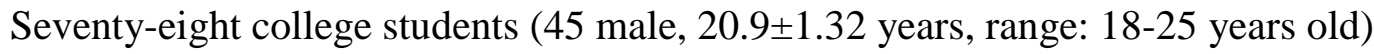


completed both MSIT and resting-state scans. All participants were right-handed, and had normal or corrected-to-normal vision. None had any current or past psychiatric or neurological disease. Each participant provided written informed consent and received monetary compensation for their time and effort. The study was approved by the Institutional Review Board of Dalian University of Technology.

\subsection{Behavioral test}

Adapting the MSIT from Bush et al.[2], each stimulus was consisted of a set of three characters (the digits "1", "2", or " 3 ", or a letter " $x ")$ and presented in the center of the screen for $1750 \mathrm{~ms}$. Among the three characters, one digit was always different from the other two digits or letters, and the participants were instructed to recognize such digit. A numeric keypad, which featured the digit "1", "2", "3" from left to right, was used as the response apparatus. The participants were asked to respond the target digits and press the corresponding numeric key by using the index, middle and ring fingers of their right hand.

During the experiment, the stimuli with two kinds of control/interference conditions appeared in a pseudorandom sequence. In the control condition, the digit was the target item and the letter " $\mathrm{X}$ " was the distractor. The size of target digit was a slightly larger than that of the distractor. The position of the presenting target digit was compatible with its position on the butter-press (e.g., "XX3", target is " 3 ", and the button be pressed at the 3rd position). In contrast, during the interference condition (integrated with Stroop, Simon and flanker effects), both the target and distractor were digits. The size of the target was sometimes larger and sometimes 
smaller than that of the distractor. The presenting target digit did not match its position on the response key (e.g., " 311 ", target is "3", but the correct key should be pressed at the 3rd position).

The MSIT was conducted individually using E-Prime software on a laptop computer in a quiet room of the lab. After instructions and practice, participants would complete three blocks of MSIT, 192 trials per block (96 control trails and 96 interference trials), for a total 576 trials. In between the blocks, the participants could take a short break. Both response accuracy and speed were equally stressed. Both reaction time (RT) and accuracy were collected for all subjects using E-Prime software. The RTs for incorrect or null responses were discarded from further analysis. According to the initial studies on MSIT[2,3], the interference effect is the difference score between RTs in the interference condition and RTs in the control condition (RTs interference-RTs control).

\subsection{Image acquisition}

MRI data were collected using a 3T Philips Achieva scanner (Philips Medical Systems, Best, Netherlands) at Dalian Municipal Central Hospital. Foam pads were used to minimize head movement. The participants were instructed to fixate on a white crosshair centered on a black background and not to sleep or think about anything in particular. The RS-fMRI scans were obtained using an echo-planar imaging sequence (TR,2000 ms; TE,30 ms; matrix, $64 \times 64 \times 36$; voxel size, $3.59 \times$ $3.59 \times 4.00 \mathrm{~mm}$; flip angle, $80^{\circ}$ ). Each RS-fMRI session lasted for 6 minutes. In addition, T1-weighted anatomical images (TR,7ms; TE,3.20ms; matrix,256×256× 
160; voxel size, $1 \times 1 \times 1 \mathrm{~mm}$; flip angle, $\left.8^{\circ}\right)$ were acquired.

The sequence of the MSIT and the imaging collection varied across subjects depending on their convenience and the fMRI scanner's schedule. Given that the cognitive task could impact on the resting-state scan [32], we tried to avoid such influence. For most of the subjects (60/78), the MSIT was conducted at least one day before the fMRI data collection. For sixteen subjects, the MSIT was performed at least one day after the fMRI data collection. For two subjects, the imaging data preceded the MSIT on the same day.

\subsection{RS-fMRI Data preprocessing}

RS-fMRI data preprocessing was performed using Data Processing Assistant for Resting-State fMRI Advanced Edition (DPARSFA V2.3, http://rfmri.org/dparsf_v2_3) based on SPM8 (http://www.fil.ion.ucl.ac.uk/spm/) [33]. The first five scans were discarded for signal equilibrium. The remaining 175 scans were preprocessed, which included the following steps: (1) slice timing correction; (2) realignment; (3) the T1-weighted anatomical images were co-registered to EPI images and segmented into gray matter (GM), white matter (WM) and cerebrospinal fluid (CSF); (4) in order to decrease the effects of physiological processes and head motion, 26 nuisance covariates (signals from WM, CSF, and Friston 24 motion parameters) were regressed out and the linear trends were removed. It should be noted that the removal of the global signal was not performed as the global signal regression in the preprocessing step is still debatable [34-36]; (5) the functional images were spatially normalized to Montreal Neurological Institute $(\mathrm{MNI})$ space (voxel size: $3 \times 3 \times 3$ ), and then 
smoothed with a Gaussian kernel with full-width at half-maximum of $4 \mathrm{~mm}$.

\subsection{Assessment of head motion}

The mean framewise displacement (FD), which obtained from Jenkinson's relative root mean square algorithm was calculated for each participant [37]. Three participants with mean FD exceeding 2 SD of the sample mean FD (threshold: 0.1688 ) were excluded $[38,39]$ and seventy-five participants were included for further analysis.

\subsection{Calculation of fALFF}

In each voxel of the brain, fALFF was computed as the ratio between the total amplitudes across $0.01-0.08 \mathrm{~Hz}$ and the sum of amplitudes across the whole frequency domain (0-0.25Hz) using DPARSFA V2.3 [24]. The individual-level voxel-wise fALFF maps were scaled by the global mean fALFF value to standardize data across participants.

\subsection{Partial correlation analysis}

Previous research have indicated that gender- and age- related differences affect the structural brain asymmetry [40] and anatomical connectivity [41]. For instance, male brains have been reported to be more asymmetric than female brains [40], and male has exhibited a lower overall cortical connectivity than female [41]. The overall cortical connectivity has been shown to decline with aging [41]. Thus, gender and age were included as covariates of no interest. Meanwhile, to further regress out the effect of head motion $[39,42]$, mean FD was also included as covariates of no interest. Taken together, partial correlation analysis was applied to remove the effects of age, 
gender and mean FD. Such analysis method was also employed to explore the relationship between ALFF and executive control effect [43].

By using Resting state fMRI Data Analysis Toolkit (REST, http://restfmri.net/forum/REST_V1.8) [44], we conducted partial correlation between fALFF and interference effect, between fALFF and RT in the interference condition, or between fALFF and RT in the control condition with controlling for age, gender and mean FD. All the correlation results were set at a threshold of corrected cluster $\mathrm{p}$ $<0.001$ (single voxel $\mathrm{p}<0.05$, cluster size $\geq 3969 \mathrm{~mm}^{3}$ ), using AlphaSim program in REST.

\section{Results}

\subsection{Behavioral results}

Mean RT and accuracy for each condition (control and interference) were calculated. Error trials were excluded from the RT analyses. The difference of RTs between both conditions for each participants (RT interference - RT control) was calculated as the interference effect (Mean $\pm \mathrm{SD}=168.14 \pm 43.26)$ [3]. In addition, the differences between control and interference conditions for RT and accuracy were assessed. The mean RT of the control trials was significantly faster than that of the interference trials by paired-t-test $\left(707.75 \pm 91.83 \mathrm{~ms}\right.$ vs. $\left.875.89 \pm 100.77 \mathrm{~ms}, \mathrm{t}=-33.66, \mathrm{p}=1.31 \times 10^{-46}\right)$. The accuracy rate between control and interference conditions was significant by Wilcoxon signed ranks test $\left(0.99 \pm 0.01\right.$ vs. $\left.0.94 \pm 0.04, \mathrm{Z}=-7.39, \mathrm{p}=-1.51 \times 10^{-13}\right)$. The descriptive statistics for accuracy and interference effect were presented (Fig. 1). 


\subsection{Brain-behavior correlations}

The significant negative fALFF-interference effect correlations were found in orbital prefrontal cortex (OPFC) and right inferior frontal cortex (IFC) at a threshold of $\mathrm{p}<$ 0.001(AlphaSim corrected) (Fig. 2, Table 1) after controlling for the effects of age, gender and mean FD. But there were no significant correlations between fALFF and interference condition, or between fALFF and control condition at a threshold of $\mathrm{p}<$ 0.001(AlphaSim corrected).

\section{Discussion}

MSIT mixing with Stroop, Simon and flanker effects were employed to assess the cognitive interference effect, which was produced by RT difference between control and interference conditions. To evaluate the cortical basis of inter-subject difference in the cognitive interference effect, the present study conducted a partial correlation analysis between fALFF and interference effect (while controlling for age, gender and mean FD). The results demonstrated significant negative relationship between fALFF and interference effect both in OPFC and right IFC; participants who induced higher fALFF in the two brain regions tended to show lower interference effect. In addition, the partial correlation analyses were performed between fALFF and RT in control condition, or between fALFF and RT in interference condition (while controlling for age, gender and mean FD), while no significant associations were found. All these results suggested that intrinsic brain activity in OPFC and right IFC is associated with the cognitive interference effect in MSIT. The current findings highlight the important role of intrinsic brain dynamic in conflict processing efficiency. 
FALFF reflects the reliable property of LFO amplitude and is more sensitive and precise in recognizing the brain activity $[24,25]$. FALFF in OPFC exhibited a significant negative correlation with interference effect in the present study, thereby suggesting that LFO amplitude in OPFC is related to processing efficiency of conflict resolution. The OPFC is functionally implicated in cognitive inhibition. People with psychiatric disorder such as OCD, behavioral variant frontotemporal dementia (bvFTD), and online gaming addiction (OGA), which are related to OPFC dysfunction, showed to impair cognitive inhibition [45-50]. In the neuroimaging studies of OCD patients, the response inhibition related to activity in right OPFC was inversely associated with the OCD symptom severity [45] and the OCD patients showed decrease activation in OPFC during Go/NO-Go and switch tasks [46]. Patients with bvFTD were showed to be linked to levels of disinhibition [47] and worse performance in the flanker task than normal controls [48]. Yuan et al. found that ALFF values of left medial OPFC in young participants with OGA were positively associated not only with the duration of OGA, but also with response errors in color-word Stroop task [49]. In Yuan et al.'s another study in young participants with OGA, there was a negative correlation between cortical thickness in the left OPFC and Stroop task response errors [50]. Similarly, patients with medial OPFC lesion also demonstrated much poorer performance on the Stroop test than patients without such lesions and normal controls [51]. Even in the normal participants, the significant activation of anterior lateral OPFC was found during response inhibition [52].Taken together, all of these results can be interpreted as that OPFC is the essence of 
inhibitory control.

On the other hand, since OPFC is anatomically connected to higher sensory, limbic and other prefrontal areas, it can integrate both multisensory and emotional information, as well as process value, so as to flexibly adapt the changing situations $[53,54]$. For instance, OPFC contributes to decision-making and action selection on the basis of encoding predicted reward information [55]. In a fMRI study of the reward-based decision-making task, Hare et al. found that activation in the medial OPFC is associated with the goal value signals, and activation in the central OPFC is related to the decision value computations [56]. Another finding reported that patient with OPFC lesion failed to flexibly inhibit existing stimulus-outcome associations in the probabilistic learning task, indicating that OPFC is important for adaptively processing outcome value in a variable situation [57]. Moreover, even without distinct rewards, the activation of medial OPFC in the current trial could predict the response speed in the next trial in a classical Stroop task, and this reflected that OPFC can impact future behavioral response [58]. Consequently, the role of OPFC is not limited to inhibitory control, but can extend to generate adaptive performance by encoding goal-directed information. The interference effect in the present study reflects the process efficiency of conflict resolution, which encompasses recognizing the target and distractor, suppressing an improper response and executing a correct response [1].A significant negative correlation between fALFF values in OPFC and interference effect on MSIT was observed, thereby indicating that OPFC function in resting-state may be strongly related to inhibitory control and flexibly modify 
behavior.

Another significant reverse association observed between fALFF and interference effect was located in right IFC, which means that the higher the efficacy of conflict resolution, the higher the fALFF in right IFC. Many neuroimaging studies of the interference tasks contribute strong evidence that the right IFC is like a brake and focuses on restraining inappropriate response impulses $[59,60]$. In stop signal task and GO/No-Go task, activation of right IFC is elevated when stop trials are successful inhibited $[1,59,60]$. Right IFC also showed significantly higher activation in conflict trials compared with nonconflict trials in the numerical contrasts of numerical Stroop task [61]. In a cueing version of Simon task, the adroitness of selective response suppression could predict the activation of right IFC and had a tight relationship with diffusion tensor imaging parameters within right IFC [62]. In agreement with these neuroimaging findings of cognitive interference resolution tasks, a meta-analysis of neuroimaging studies of such tasks has reported that the right IFC is the critical region for interference resolution during response performance [1]. Another recent systemic research on inhibitory control, in which multiple quantitative analyses including meta-analysis were used, has revealed that the functional dissociation between anterior insula and right IFC: anterior insula is related to the silent processing, whereas right IFC is associated with inhibitory control [63]. Moreover, although some have proposed that right IFC is merely involved in monitoring context [64], the finding by Wessel et al. was not in line with such notion and supported the association between right IFC and inhibitory processes [65]. In Wessel et al.'s study, they found 
that direct electrical stimulation of right IFC decelerated Go-trial response (in comparison with control-site stimulation) and that right IFC stimulation elicited greater deceleration when motor brake was needed in "Maybe Stop" condition compared with the "No Stop" condition. Recently, researchers addressed the argument about the right IFC role in monitoring context and emphasized right IFC role in inhibitory control [66]. Finally, similar notion supporting the inhibitory function of right IFC have been found in the RS-fMRI studies. For instance, the regional homogeneity of RS-fMRI signals in right IFC exhibited a significant association with the SSRT in the stop signal task [67]. Another RS-fMRI study, which explored the intrinsic brain activity, could predict the executive control effect and showed a significant negative correlation between behavior results of the executive control effect in attention network task and the ALFF in right precuneus [43]. The right IFC is functionally connected with the right precunues and their connectivity strength is negatively associated with the executive control ratio scores [43]. The result of our study is in accord with these findings and further indicates that the resting state activity in right IFC could predict the inhibitory processing of interference resolution.

Interestingly, the fALFF in OPFC and right IFC, which exhibited significant correlation with interference effect in current RS-fMRI study, seemed not to be usually activated by MSIT task. In previous MSIT-related fMRI analysis, the aforementioned CFP network was strongly activated when comparing between interference condition and control condition $[7,8]$. Evidently, the fALFF-behavior correlation analysis and MSIT-related fMRI analysis reflect two distinct brain 
mechanisms of conflict resolution processes. Accordingly, the activation of the CFP network identifies and characterizes the brain processing of conflict resolution, and the fALFF of RS-fMRI signals in OPFC and right IFC might predict the performance of conflict resolution.

FALFF of OPFC and right IFC, which negatively correlated with conflict effect of MSIT in our study, manifested that these two regions are related to adaptive performance and effective inhibition in conflict resolution. It further confirmed that fALFF of prefrontal cortex (PFC) could be an important index to predict the inter-individual changes in self-control. The findings are verified by other studies on fALFF of PFC and have observed that the fALFF in anterior cingulate and PFC (including medial PFC, IFC and ventrolateral PFC) was obviously elevated in smokers after receiving 5-hour meditation training [68], which manifested that meditation practice can enhance smoker's self-regulation ability and decrease their craving for smoking. In another study, compared with healthy subjects, major depressive disorder patients exhibited lower fALFF in PFC, covering dorsolateral PFC and OPFC, which were interpreted as the important brain region for cognitive control function [69]. In conclusion, further research is needed to clarify the relationship between fALFF of PFC and self-control. In addition, fALFF of OPFC and right IFC could serve as biomarkers for evaluating individual's capability of self-control and conflict resolution.

Finally, it is necessary to consider some limitations in the current study and highlight opportunities for further studies. The findings of our study depended on the 
measure of fALFF of RS-fMRI. Prior studies as mentioned in the introduction have investigated the relationship between MSIT-evoked activation and resting-state [12, 16, 20]. Besides, by utilizing MSIT-related fMRI and structural analysis, Salami et al. found that age-related grey matter losses in anterior brain areas were related to the enhanced interference resolution in the young group [70]. All these findings indicate that future study could use MSIT-related fMRI, RS-fMRI and structure fMRI and incorporate these multimodal image features to further characterize the structural and functional interference effect on MSIT.

\section{Conclusions}

In summary, using fALFF-interference effect of MSIT correlation analysis, we firstly observed that the fALFF of OPFC and right IFC are significantly and negatively correlated with the interference effect of MSIT. These findings provide a new horizon for understanding the neural mechanism of processing efficiency of conflict resolution and cognitive control.

\section{Acknowledgements}

We thank lab menbers for assistance with RS-fMRI data collection. This work was supported by the Office of Naval Research.

\section{References}

[1] Nee DE, Wager TD, Jonides J. Interference resolution: insights from a meta-analysis of neuroimaging tasks. Cogn Affect Behav Neurosci 2007; 7: 1-17. 
[2] Bush G, Shin LM, Holmes J, Rosen BR, Vogt BA. The Multi-Source Interference Task: validation study with fMRI in individual subjects. Mol Psychiatry 2003; 8: 60-70.

[3] Bush G, Shin LM. The Multi-Source Interference Task: an fMRI task that reliably activates the cingulo-frontal-parietal cognitive/attention network. Nat Protoc 2006; 1:308-13.

[4] Bush G, Vogt BA, Holmes J, Dale AM, Greve D, Jenike MA, et al. Dorsal anterior cingulate cortex: a role in reward-based decision making. Proc Natl Acad Sci U S A 2002; 99: 523-8.

[5] Sheth SA, Mian MK, Patel SR, Asaad WF, Williams ZM, Dougherty DD, et al. Human dorsal anterior cingulate cortex neurons mediate ongoing behavioural adaptation. Nature 2012; 488: 218-21.

[6] Zanto TP, Gazzaley A. Fronto-parietal network: flexible hub of cognitive control. Trends Cogn Sci 2013; 17: 602-3.

[7] Bush G. Cingulate, frontal, and parietal cortical dysfunction in attention-deficit/hyperactivity disorder. Biol Psychiatry 2011; 69: 1160-7.

[8] Heckers S, Weiss AP, Deckersbach T, Goff DC, Morecraft RJ, Bush G. Anterior cingulate cortex activation during cognitive interference in schizophrenia. Am $\mathbf{J}$ Psychiatry 2004; 161: 707-15.

[9] Yücel M, Lubman DI, Harrison BJ, Fornito A, Allen NB, Wellard RM, et al. A combined spectroscopic and functional MRI investigation of the dorsal anterior cingulate region in opiate addiction. Mol Psychiatry 2007; 12:691-702. 
[10] Harrison BJ, Yücel M, Fornito A, Wood SJ, Seal ML, Clarke K, et al.

Characterizing anterior cingulate activation in chronic schizophrenia: a group and single-subject fMRI study. Acta Psychiatr Scand 2007; 116: 271-9.

[11] Yücel M, Harrison BJ, Wood SJ, Fornito A, Wellard RM, Pujol J, et al. Functional and biochemical alterations of the medial frontal cortex in obsessive-compulsive disorder. Arch Gen Psychiatry 2007; 64: 946-55.

[12] Fitzgerald KD, Stern ER, Angstadt M, Nicholson-Muth KC, Maynor MR, Welsh RC, et al. Altered function and connectivity of the medial frontal cortex in pediatric obsessive-compulsive disorder. Biol Psychiatry 2010; 68: 1039-47.

[13] Brown AB, Biederman J, Valera EM, Doyle AE, Bush G, Spencer T, et al. Effect of dopamine transporter gene (SLC6A3) variation on dorsal anterior cingulate function in attention-deficit/hyperactivity disorder. Am J Med Genet B Neuropsychiatr Genet 2010; 153B: 365-75.

[14] Shin LM, Bush G, Milad MR, Lasko NB, Brohawn KH, Hughes KC, et al. Exaggerated activation of dorsal anterior cingulate cortex during cognitive interference: a monozygotic twin study of posttraumatic stress disorder. Am J Psychiatry 2011; 168: 979-85.

[15] Harding IH, Solowij N, Harrison BJ, Takagi M, Lorenzetti V, Lubman DI, et al. Functional connectivity in brain networks underlying cognitive control in chronic cannabis users. Neuropsychopharmacology 2012; 37: 1923-33.

[16] Davey CG, Yücel M, Allen NB, Harrison BJ. Task-related deactivation and functional connectivity of the subgenual cingulate cortex in major depressive 
disorder. Front Psychiatry 2012;3:14.

[17] Matthews SC, Simmons AN, Strigo I, Jang K, Stein MB, Paulus MP

Heritability of anterior cingulate response to conflict: an fMRI study in female twins. Neuroimage 2007; 38: 223-7.

[18] Fitzgerald KD, Perkins SC, Angstadt M, Johnson T, Stern ER, Welsh RC, et al. The development of performance-monitoring function in the posterior medial frontal cortex. Neuroimage 2010; 49: 3463-73.

[19] Shehzad Z, DeYoung CG, Kang Y, Grigorenko EL, Gray JR. Interaction of COMT val 158 met and externalizing behavior: relation to prefrontal brain activity and behavioral performance. Neuroimage 2012; 60:2158-68.

[20] Dwyer DB, Harrison BJ, Yücel M, Whittle S, Zalesky A, Pantelis C, et al. Large-Scale brain network dynamics supporting adolescent cognitive control. J Neurosci 2014; 34:14096-107.

[21] Fox MD, Raichle ME. Spontaneous fluctuations in brain activity observed with functional magnetic resonance imaging. Nat Rev Neurosci 2007; 8: 700-11.

[22] Raichle ME, Mintun MA. Brain work and brain imaging. Annu Rev Neurosci 2006; 29:449-76.

[23] Raichle ME. Two views of brain function. Trends Cogn Sci 2010;14:180-90.

[24] Zou QH, Zhu CZ, Yang Y, Zuo XN, Long XY, Cao Q, et al. An improved approach to detection of amplitude of low-frequency fluctuation (ALFF) for resting-state fMRI: fractional ALFF. J Neurosci Methods 2008; 172: 137-41.

[25] Zuo XN, Di Martino A, Kelly C, Shehzad ZE, Gee DG, Klein DF, et al. The oscillating brain: complex and reliable. Neuroimage 2010; 49: 1432-45. 
[26] Kunisato Y, Okamoto Y, Okada G, Aoyama S, Nishiyama Y, Onoda K, et al. Personality traits and the amplitude of spontaneous low-frequency oscillations during resting state. Neurosci Lett 2011; 492: 109-13.

[27] Wei L, Duan X, Zheng C, Wang S, Gao Q, Zhang Z, et al. Specific frequency bands of amplitude low - frequency oscillation encodes personality. Hum Brain Mapp 2014; 35: 331-9.

[28] Mennes M, Zuo XN, Kelly C, Di Martino A, Zang YF, Biswal B, et al. Linking inter-individual differences in neural activation and behavior to intrinsic brain dynamics. Neuroimage 2011; 54:2950-9.

[29] Hu S, Chao HH, Zhang S, Ide JS, Li CS. Changes in cerebral morphometry and amplitude of low-frequency fluctuations of BOLD signals during healthy aging: correlation with inhibitory control. Brain Struct Funct 2014; 219:983-94.

[30] van Dam WO, Decker SL, Durbin JS, Vendemia JM, Desai RH. Resting state signatures of domain and demand-specific memory performance. Neuroimage $2015 ; 118: 174-82$.

[31] Xu M, De Beuckelaer A, Wang X, Liu L, Song Y, Liu J. Regional amplitude of the low-frequency fluctuations at rest predicts word-reading skill. Neuroscience 2015; 298: 318-28.

[32] Andrews-Hanna JR, Reidler JS, Sepulcre J, Poulin R, Buckner RL. Functional-anatomic fractionation of the brain's default network. Neuron 2010; 65: $550-62$.

[33] Chao-Gan Y, Yu-Feng Z. DPARSF: a MATLAB toolbox for "pipeline” data analysis of resting-state fMRI. Front Syst Neurosci 2010; 4:13. 
[34] Murphy K, Birn RM, Handwerker DA, Jones TB, Bandettini PA. The impact of global signal regression on resting state correlations: Are anti-correlated networks introduced? Neuroimage 2009; 44: 893-905.

[35] Saad ZS, Gotts SJ, Murphy K, Chen G, Jo HJ, Martin A, et al. Trouble at rest: how correlation patterns and group differences become distorted after global signal regression. Brain Connect 2012; 2: 25-32.

[36] Keller CJ, Bickel S, Honey CJ, Groppe DM, Entz L, Craddock RC, et al. Neurophysiological investigation of spontaneous correlated and anticorrelated fluctuations of the BOLD signal. J Neurosci 2013; 33: 6333-42.

[37] Jenkinson M, Bannister P, Brady M, Smith S. Improved optimization for the robust and accurate linear registration and motion correction of brain images. Neuroimage 2002; 17: 825-41.

[38] Yan CG, Craddock RC, Zuo XN, Zang YF, Milham MP. Standardizing the intrinsic brain: towards robust measurement of inter-individual variation in 1000 functional connectomes. Neuroimage 2013; 80: 246-62.

[39] Yan C, Cheung B, Kelly C, Colcombe S, Craddock RC, Di Martino A, et al. A comprehensive assessment of regional variation in the impact of head micromovements on functional connectomics. Neuroimage 2013; 76: 183-201.

[40] Kovalev VA, Kruggel F, von Cramon DY. Gender and age effects in structural brain asymmetry as measured by MRI texture analysis. Neuroimage 2003; 19 : 895-905.

[41] Gong G, Rosa-Neto P, Carbonell F, Chen ZJ, He Y, Evans AC. Age-and 
gender-related differences in the cortical anatomical network. J Neurosci 2009; 29:

15684-93.

[42] Power JD, Mitra A, Laumann TO, Snyder AZ, Schlaggar BL, Petersen SE.

Methods to detect, characterize, and remove motion artifact in resting state fMRI.

Neuroimage 2014; 84: 320-41.

[43] Xu J, Rees G, Yin X, Song C, Han Y, Ge H, et al. Spontaneous neuronal activity predicts intersubject variations in executive control of attention.

Neuroscience 2014; 263: 181-92.

[44] Song XW, Dong ZY, Long XY, Li SF, Zuo XN, Zhu C, et al. REST: a toolkit for resting-state functional magnetic resonance imaging data processing. PLoS One 2011; 6: e25031.

[45] Roth RM, Saykin AJ, Flashman LA, Pixley HS, West JD, Mamourian AC. Event-related functional magnetic resonance imaging of response inhibition in obsessive-compulsive disorder. Biol Psychiatry 2007; 62: 901-9.

[46] Page LA, Rubia K, Deeley Q, Daly E, Toal F, Mataix-Cols D, et al. A functional magnetic resonance imaging study of inhibitory control in obsessive-compulsive disorder. Psychiatry Res 2009; 174: 202-9.

[47] Hornberger M, Geng J, Hodges JR. Convergent grey and white matter evidence of orbitofrontal cortex changes related to disinhibition in behavioural variant frontotemporal dementia. Brain 2011; 134: 2502-12.

[48] Krueger CE, Bird AC, Growdon ME, Jang JY, Miller BL, Kramer JH. Conflict monitoring in early frontotemporal dementia. Neurology 2009; 73: 349-55. 
[49] Yuan K, Jin C, Cheng P, Yang X, Dong T, Bi Y, et al. Amplitude of low frequency fluctuation abnormalities in adolescents with online gaming addiction. PLoS One 2013; 8: e78708.

[50] Yuan K, Cheng P, Dong T, Bi Y, Xing L, Yu D, et al. Cortical thickness abnormalities in late adolescence with online gaming addiction. PLoS One 2013; 8: e53055.

[51] Szatkowska I, Szymańska O, Bojarski P, Grabowska A. Cognitive inhibition in patients with medial orbitofrontal damage. Exp Brain Res 2007; 181: 109-15.

[52] Horn NR, Dolan M, Elliott R, Deakin JF, Woodruff PW. Response inhibition and impulsivity: an fMRI study. Neuropsychologia 2003; 41: 1959-66.

[53] Elliott R, Deakin B. Role of the Orbitofrontal Cortex in Reinforcement Processing and Inhibitory Control: Evidence from functional magnetic resonance imaging Studies in Healthy Human Subjects. In: Bradley R, Harris RA, Jenner P, editors. International Review of Neurobiology (Volume 65), Academic Press; 2005, p. 89-116.

[54] Gottfried JA, O'Doherty J, Dolan RJ. Encoding predictive reward value in human amygdala and orbitofrontal cortex. Science 2003; 301: 1104-7.

[55] Frank MJ, Claus ED. Anatomy of a decision: striato-orbitofrontal interactions in reinforcement learning, decision making, and reversal. Psychol Rev 2006; $113: 300-26$.

[56] Hare TA, O'Doherty J, Camerer CF, Schultz W, Rangel A. Dissociating the role of the orbitofrontal cortex and the striatum in the computation of goal values and 
prediction errors. J Neurosci 2008; 28: 5623-30.

[57] Tsuchida A, Doll BB, Fellows LK. Beyond reversal: a critical role for human orbitofrontal cortex in flexible learning from probabilistic feedback. J Neurosci 2010; 30: 16868-75.

[58] Verstynen TD. The organization and dynamics of corticostriatal pathways link the medial orbitofrontal cortex to future behavioral responses. J Neurophysiol 2014; 112: 2457-69.

[59] Aron AR, Robbins TW, Poldrack RA. Inhibition and the right inferior frontal cortex. Trends Cogn Sci 2004; 8: 170-7.

[60] Aron AR, Robbins TW, Poldrack RA. Inhibition and the right inferior frontal cortex: one decade on. Trends Cogn Sci 2014; 18: 177-85.

[61] Tang J, Critchley HD, Glaser DE, Dolan RJ, Butterworth B. Imaging informational conflict: a functional magnetic resonance imaging study of numerical Stroop. J Cogn Neurosci 2006; 18: 2049-62.

[62] Forstmann BU, Jahfari S, Scholte HS, Wolfensteller U, van den Wildenberg WP, Ridderinkhof KR. Function and structure of the right inferior frontal cortex predict individual differences in response inhibition: a model-based approach. $\mathbf{J}$ Neurosci 2008; 28:9790-6.

[63] Cai W, Ryali S, Chen T, Li CS, Menon V. Dissociable Roles of Right Inferior Frontal Cortex and Anterior Insula in Inhibitory Control: Evidence from Intrinsic and Task-Related Functional Parcellation, Connectivity, and Response Profile Analyses across Multiple Datasets. J Neurosci 2014; 34:14652-67. 
[64] Munakata Y, Herd SA, Chatham CH, Depue BE, Banich MT, O’Reilly RC. A unified framework for inhibitory control. Trends Cogn Sci 2011; 15: 453-9.

[65] Wessel JR, Conner CR, Aron AR, Tandon N. Chronometric electrical stimulation of right inferior frontal cortex increases motor braking. J Neurosci $2013 ; 33: 19611-9$.

[66] Aron AR, Robbins TW, Poldrack RA. Right inferior frontal cortex: addressing the rebuttals. Front Hum Neurosci 2014; 8:905.

[67] Tian L, Ren J, Zang Y. Regional homogeneity of resting state fMRI signals predicts stop signal task performance. Neuroimage 2012; 60: 539-44.

[68] Tang YY, Tang R, Posner MI. Brief meditation training induces smoking reduction. Proc Natl Acad Sci USA 2013; 110:13971-5.

[69] Wang L, Dai W, Su Y, Wang G, Tan Y, Jin Z, et al. Amplitude of low-frequency oscillations in first-episode, treatment-naive patients with major depressive disorder: a resting-state functional MRI study. PLoS One 2012; 7: e48658.

[70] Salami A, Rieckmann A, Fischer H, Bäckman L. A multivariate analysis of age-related differences in functional networks supporting conflict resolution. Neuroimage 2014; 86:150-63. 


\section{Figure Captions}

\section{Figure 1.}

Descriptive statistics for MSIT behavioral data. (A) Mean accuracy rate in interference condition (left) and control condition (right). The black dots indicate mean accuracy rates; the black lines connect the dots corresponding to the same individual. (B) Mean interference effect. Error bars indicate standard error of the means.

\section{Figure 2.}

Significant correlations of fALFF and interference effect in OPFC (A) and right IFC (B). Blue indicates negative correlations. The figures below each image are the Montreal Neurological Institute X-Y- Z coordinates. The threshold was p $<0.001$ (AlphaSim-corrected). An R-value scale is exhibited below the two figures. Abbreviations: $\mathrm{OPFC}=$ orbital prefrontal cortex; IFC $=$ inferior frontal cortex. 

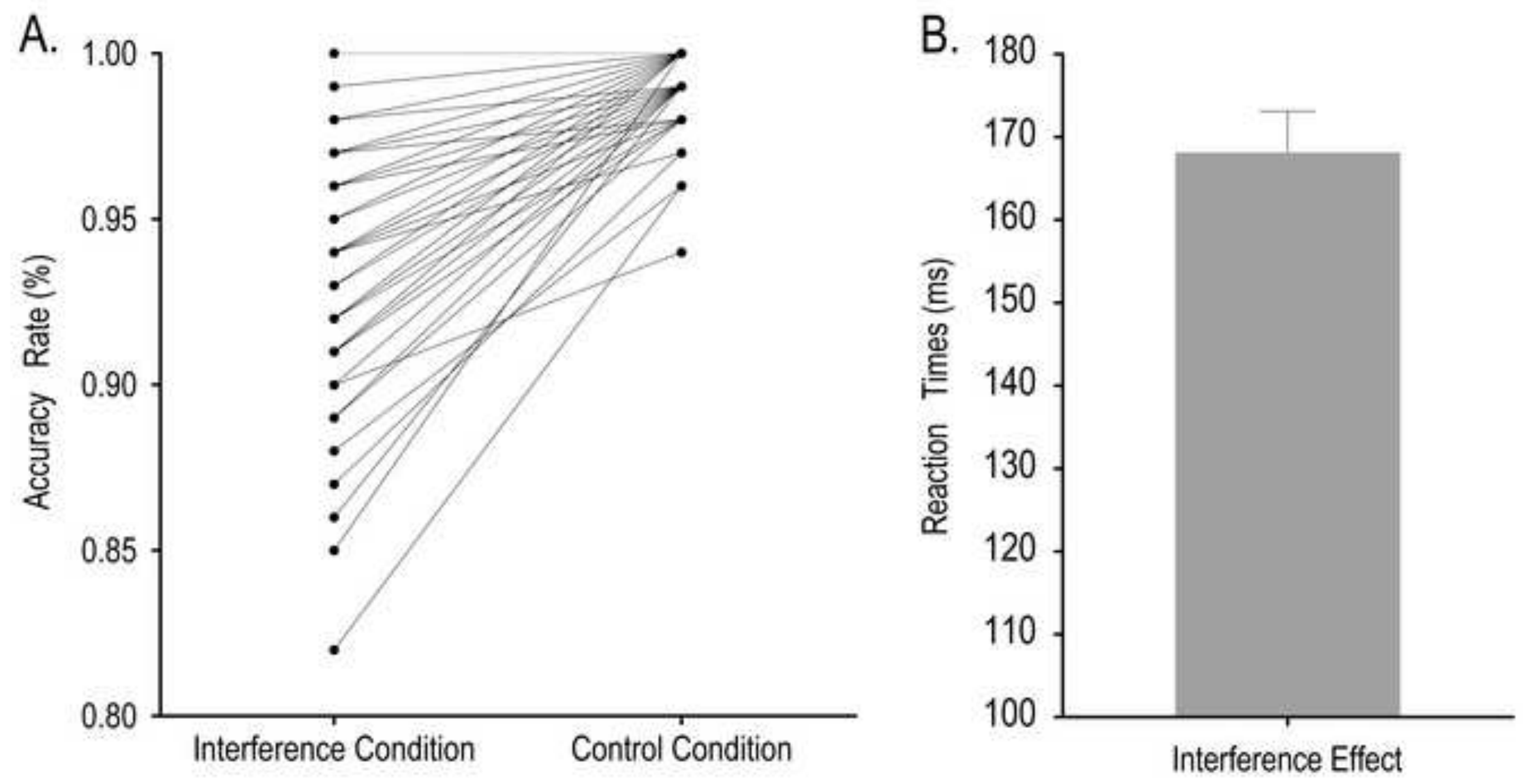
A. OPFC
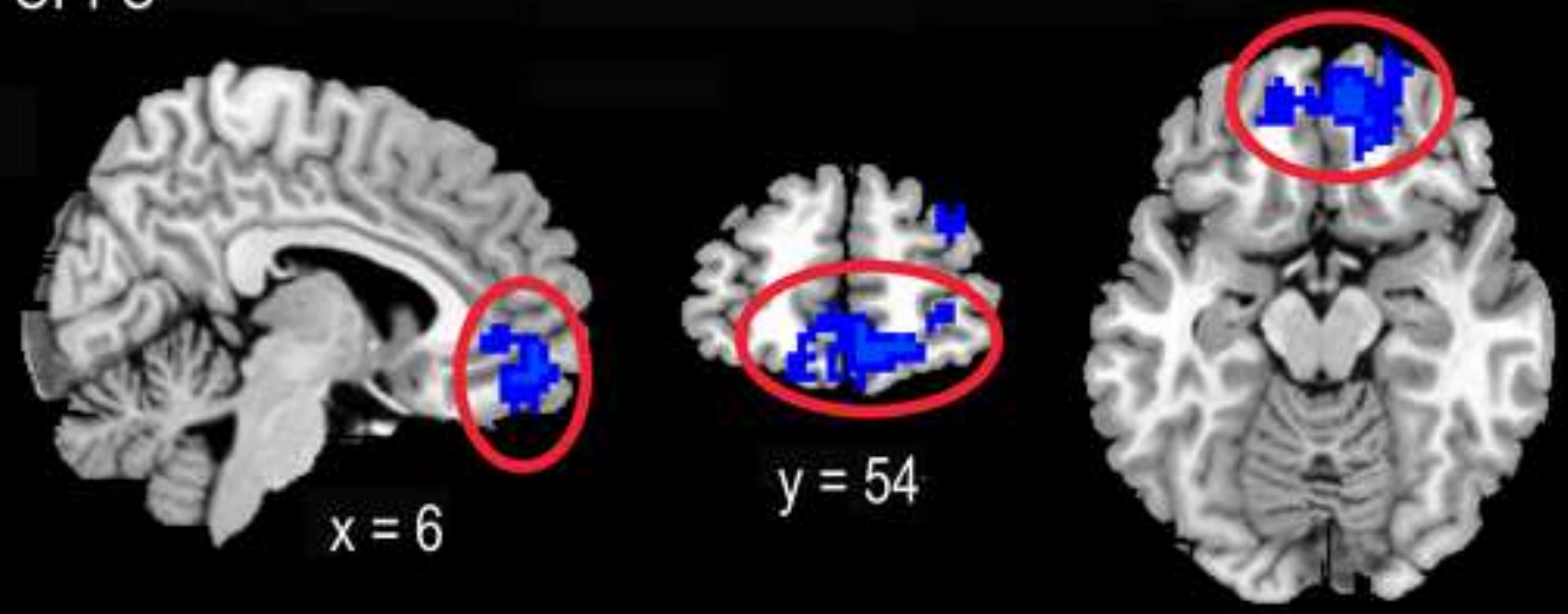

$$
z=-15
$$

B. right IFC
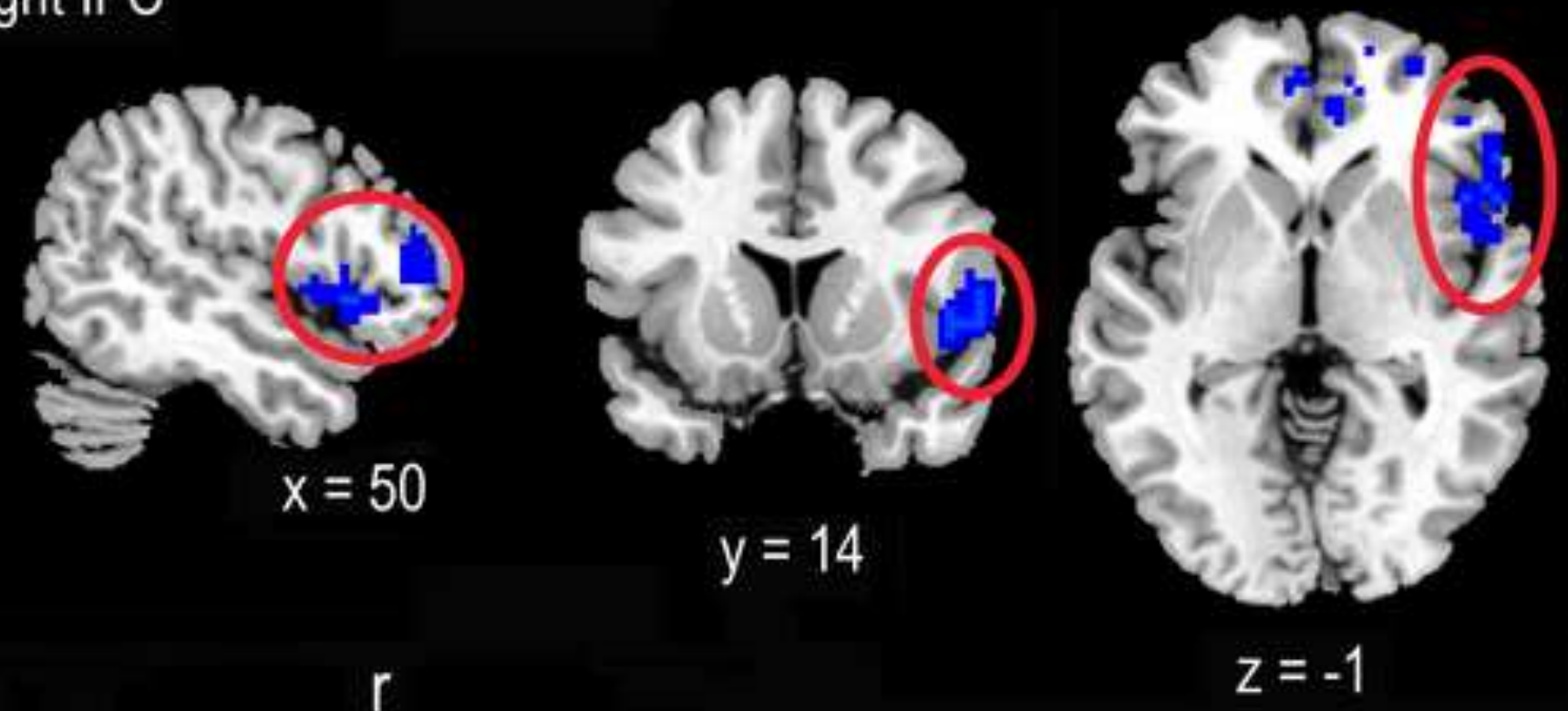

\begin{tabular}{ll|l|r}
-0.23 & $\mid$ & I & |
\end{tabular} 
Table 1.

Significant fALFF-interference effect correlations across subjects.

\begin{tabular}{|c|c|c|c|c|c|c|}
\hline \multirow[t]{2}{*}{ Region } & \multirow[t]{2}{*}{$\mathrm{BA}$} & \multirow[t]{2}{*}{$\begin{array}{l}\text { Cluster size } \\
\quad\left(\mathrm{mm}^{3}\right)\end{array}$} & \multirow[t]{2}{*}{ MNI } & \multicolumn{2}{|c|}{$\begin{array}{l}\text { coordinate } \\
\text { eak) }\end{array}$} & \multirow[t]{2}{*}{$\begin{array}{c}\mathrm{r} \\
\text { (peak) }\end{array}$} \\
\hline & & & & $\mathrm{y}$ & $\mathrm{Z}$ & \\
\hline OPFC & $10 / 11$ & 10989 & 6 & 54 & -9 & -0.42 \\
\hline IFC_R & $45 / 46 / 47 / 48$ & 7803 & 42 & 33 & 21 & -0.52 \\
\hline
\end{tabular}

Abbreviations: $\mathrm{MNI}=$ Montreal Neurological Institute; $\mathrm{BA}=$ Brodmann's area; OPFC $=$ orbital prefrontal cortex; IFC= inferior frontal cortex; $\mathrm{R}=$ Right.

The threshold was $\mathrm{p}<0.001$ (AlphaSim-corrected). 